Sādhanā Vol. 39, Part 5, October 2014, pp. 1095-1117. (C) Indian Academy of Sciences

\title{
Information extraction from topographic map using colour and shape analysis
}

\author{
NIKAM GITANJALI GANPATRAO* and \\ JAYANTA KUMAR GHOSH
}
Geomatics Engineering Group, Civil Engineering Department, Indian Institute of Technology, Roorkee, 247667 India
e-mail: chalakgeogh@yahoo.in; gjkumfce@yahoo.com

MS received 21 July 2013; revised 19 March 2014; accepted 14 April 2014

\begin{abstract}
The work presented in this paper is related to symbols and toponym understanding with application to scanned Indian topographic maps. The proposed algorithm deals with colour layer separation of enhanced topographic map using kmeans colour segmentation followed by outline detection and chaining, respectively. Outline detection is performed through linear filtering using canny edge detector. Outline is then encoded in a Freeman way, the $\mathrm{x}-\mathrm{y}$ offsets have been used to obtain a complex representation of outlines. Final matching of shapes is done by computing Fourier descriptors from the chain-codes; comparison of descriptors having same colour index is embedded in a normalized scalar product of descriptors. As this matching process is not rotation invariant (starting point selection), an interrelation function has been proposed to make the method shifting invariant. The recognition rates of symbols, letters and numbers are 84.68, 91.73 and $92.19 \%$, respectively. The core contribution is dedicated to a shape analysis method based on contouring and Fourier descriptors. To improve recognition rate, obtaining most optimal segmentation solution for complex topographic map will be the future scope of work.
\end{abstract}

Keywords. Colour segmentation; shape analysis; outline detection; chain coding; template matching.

\section{Introduction}

Scanning of topographic map provides a comprehensive digital repository of topographic maps developed by Survey of India (SOI), but it is not in computer understandable format. Government and agencies capture digital information or convert existing analogous map information. For instance, maps available in a vectorized format and organized in layers are often insufficient to recognize geographical objects relevant for a certain application. This deficiency is even more evident for patterns of geographical objects interesting for geographers, geologists and

*For correspondence 
town planners. Beside the task of solving spatial problems, automatic map understanding can be used to support other applications, like map generalization (Zhang 2009), spatial datasets matching (Volz \& Walter 2006; Butenuth et al. 2007; Chen \& Walter 2009), data fusion (Wiemann \& Bernard 2010; Anders \& Fritsch 1996) or data update (Walter 2004; Malerba et al. 2003). Furthermore, automatic map understanding can support data acquisition processes or used to form valuable set of information for large scale map analysis. Therefore, it is an interesting scope for an automated understanding of map to automate the extraction of information from unintelligent raster topographic map. The study is based on the assumption that the map understanding process typically follows computer vision technique driven by intelligent method that deciphers objects based on similarity criteria. The objective of this paper is to explain system development for understanding symbols, letters and numbers from scanned topographic map by applying shape description scheme to determine the interconnected trends and patterns and to find templates based on similarity criteria. Shape representation and description scheme with auto and inter correlation functions which are invariant to size and orientation is proposed. Our earlier work on legend understanding (Nikam \& Ghosh 2012) discusses FFBN architecture for structure primitives' recognition. However, the issues like orientation of symbols and highly interconnected nature of topographic map come into picture while building the complete integrated solution for map understanding which is not addressed in our earlier work. In this paper, complete solution that can understand symbols, letters and numbers appearing on a map is presented. Two main issues raised out of that initial work are the choice of an appropriate set of features to be used for the symbol representation or description along with colour as it is most dominant and distinguishing feature in raster map and the definition of computational methods for their interpretation. In this work, we investigate these two issues, i.e., symbol description and computational methods for the interpretation of geometrical attribute like colour and shape feature. Psychological studies (Biederman \& Ju 1988) prove that humans can recognize object using just the outline (shape) which carries characteristic information about it. A symbol is an essential component of a topographic map and its recognition based on its shape, is important in map understanding system. Also, letters and numbers on topographic maps provide additional information about the terrain. Contribution from this paper includes topographic map symbol and character understanding based on shape analysis and template matching technique.

Section 2 briefly reviews earlier work, while section 3 describes colour segmentation method applied. Section 4 gives detailed description of shape analysis techniques and similarity measures which are used for template matching. In section 5, the methodology used for creating the understanding, image pre-processing, primitive recognition, template generation and matching are described. Section 6 reports the experimental results. Finally, section 7 includes conclusion.

\section{Review of earlier work}

The work of map understanding started with a cartographic pattern recognition system using homogeneous parallel algorithms (Starr 1984; Ejiri et al. 1984), automatic map recognition system (Kasturi \& Alimony 1988) and query-driven map recognition based on template matching (Yamada et al. 1997). The method (Samet \& Soffer 1994) focused on extracting specific features such as points and lines based on a computationally intensive multi-angled parallelism method, whereas (Samet \& Soffer 1996, 1998) reported a legend driven map recognition system. It relies on weight bounded nearest-neighbour classifier that is noise sensitive and needs separate map layers as inputs. Due to the large variety of the available feature extraction methods 
(Trier et al. 1996; Heutte et al. 1993) many researchers have turned towards the use of several feature extractors with more complex structures of classification. The set of features (either statistical or structural) describing a pattern must be constrained to map the feature vector. This mapping consists in a parameterization of each feature, i.e., defining numerical parameters for each feature (Graeff \& Carosio 2002). Most often, the parameterization of such features is obtained using a zoning-like technique (Heutte et al. 1993): a fixed n X m grid is superimposed on the pattern image and the feature is searched in each of the $\mathrm{n} X \mathrm{~m}$ regions, thus giving a binary feature vector of length $\mathrm{n} X \mathrm{~m}$. Unfortunately, this results in a loss of data on the feature position additionally on a loss of continuity within the parameter area. An approach for the automatic interpretation of scanned topographic maps with query languages can be found in (Viglino \& Pierrot-Deseilligny 2003). In this, the interpretation is done with pattern recognition algorithms in the raster domain. The detected objects are implicitly contained in the raster images but were explicitly modelled when the corresponding analogue map was produced. Therefore, the objects are already visible, but cannot be queried because of the raster representation. A combination of a raster- and vectorbased approach is discussed in (Viglino \& Pierrot-Deseilligny 2003). The input for this process is a raster map that is converted into a vector representation. Different object classes (for example buildings, hangars or parcels) are reconstructed with low level primitive extraction and subsequent classification. Spatial data mining approaches are reported in (Heinzle \& Sester 2004). They describe the automatic extraction of classical metadata from spatial data sets and concepts of information retrieval to derive implicit information with data mining algorithms. Heinzle et al. (2007) continued their work and developed an algorithm for the automatic recognition of patterns in road network. The search for patterns in maps in order to detect implicit information for the automatic map generalization is described in (Mackaness \& Edwards 2002). They argue that any map can be seen as a subset of possible patterns and a map generalization is a set of transformations from one pattern to another. An ontology driven pattern recognition approach for the detection of terraced houses in vector data is presented in (Luscher et al. 2008). They use ontologies to describe the characteristics of terraced houses and map this ontology onto a pattern recognition process. Steinhauer et al. (2001) present a method for recognition of abstract regions in a map. An abstract region consists of several map objects, which are grouped to a single object. The process is subdivided into two steps. First, region candidates are selected based on an evaluation of neighbourhood relations. Then, objects which consist of a hierarchical combination of single objects are recognized with a grammar-based compiler approach. Automatic sketch interpretation is a problem which has many similarities to the problem of automatic map interpretation (Wuersch \& Egenhofer 2008). However, in sketch interpretation the main focus is more on segmentation, classification and labelling (Sezgin \& Davis 2005), whereas in map interpretation the focus is more on the following tasks, like segmentation or data mining. Maps consist typically of well-formed geometrical objects whereas sketches could also be represented by very simple geometrical entities. Nevertheless, both research areas have a large overlap. Different map objects have typical geometrical appearances depending on their object type. For example: houses have typically rectangular structures, rivers are normally represented with smooth lines and streets are often represented with straight lines. Some objects have a very typical unique appearance, like churches. In order to interpret the object type, the objects can be represented with a feature vector, which consists of different geometrical measures, and then classified with an unsupervised or supervised classification algorithm. Complex objects can also be interpreted with model-based approaches. Weindorf (2002) proposed an approach that is based on a grammatical description of objects. The model is represented with grammatical rules in PROLOG (Logic Programming Language). The inputs are geometrical primitives (lines and text elements) which are grouped together by interpreting the grammatical rules. A graph-based approach for 
detecting geometrical structures in road networks is described in (Heinzle et al. 2005, 2007). The patterns (e.g., grids, stars and rings) are used for automatic determination of city centers in vector maps. Top-down approaches are suggested to be especially suitable for recognizing symbols or letters from maps or technical drawings in (Ventura \& Schettini 1994; Myers et al. 1996; Den Hartog et al. 1996; Reiher et al. 1996). As paper focuses on understanding the pattern of symbols, letters and numbers from topographic map, we have reported research work which is done in recognition and interpretation of pattern. Traditional approach of overlying used for matching (Ebi et al. 1994) generates lots of false positives in interpretation. Research has also been done by separating the layers of scanned maps by colours as in (Roy et al. 2007; Dhar \& Chanda 2006; Chaing et al. 2005; Khotanzad \& Zink 2003). Other systems as reported in (Chaing et al. 2005; Khotanzad \& Zink 2003; Gamba \& Mecocci 1999; Wiskott \& Malsburg 1993), mostly consider the extraction of one or more types of features and primitives from maps but do not address the problems of symbols, non-horizontal text or character. Due to the problems in the extraction of features from topographic maps i.e. differential geometry and highly interconnected nature of many features, many of the systems reported in the literature have focused on maps where features are well-separated from each other or printed in separate colours (Gamba \& Mecocci 1999; Wiskott \& Malsburg 1993). These recognition schemes relied on a heuristically chosen set of features. Lam \& Suen (1988) developed a method for the recognition of handwritten numbers using structural classifier and recognition algorithm. Significant works on well-separated character recognition in the past two decades include the multi-font character recognition scheme and direction specific spatial features with structural configuration scheme (Pavlidis 1980; Kimura \& Shridhar 1991). Chen \& Lieh (1990) developed a two-layer random graph based scheme containing components and strokes as primitives and variation which is further encoded in the random graphs. Gader et al. (1991) developed template and a model matching model in two stage recognition system. Kjersti et al. (1995) developed a Hidden Markov Model for text recognition from gray level images but failed to handle problems of invariance to scale and font. Extended freeman's chain encoding schemes (Chan \& Yeung 1999) have been used to extract feature primitives of numbers and letters but suffers from directional ambiguity. Chan \& Yeung (1999) used Shape's primitive and Freeman's chain code to recognize handwritten alphanumeric characters. No work on recognition and understanding of labels in the raster topographic map has been reported so far in the literature. A method based on perception of shape i.e., outline or boundary of symbol which provides a collective understanding of size, form and orientation is required towards development of a topographic map understanding system. To cope with the above mentioned problems, authors have decided to normalize the structural features by continuous numerical variables, such as the ' $\mathrm{X}-\mathrm{Y}$ position' of exterior outline or external outline. Indeed, these variables are calculated relative to the direction of freeman chain code of the shape and normalized in complex chain code according to the width or the height of the pattern bounding box in the form of template. This allows to locate accurately the structural features and to respect the principle of continuity. Thus, main objective of this paper is to describe an algorithm which is simple and straightforward that allows efficient representation or description scheme for faster matching that is based on similarity measures.

\section{Colour segmentation}

The instinctive and normal approach to layer separation problem is to apply the R, G, B pixel values for classification. However, a direct segmentation approach is not suitable for topographic 
map segmentation due to broad variations in colour intensity. For example, the green component of red pixel may be more than the green component of green pixel in the same map. Secondly, as a priori probabilities of pixels in different layers are not comparable, the cluster corresponding to the dominant layer tends to invade the other layers.

Another difficulty is that the intensity values of black or white pixels rarely have the ideal values of $(0,0,0)$ or $(255,255,255)$ and such pixels tend to be wrongly classified. Because of the above difficulties, the strategy devised for colour segmentation first determines the initial cluster centers on the basis of an enhanced image, followed by the separation. Dhar \& Chanda (2006) have carried out layer separation by advanced k means algorithm and then recognition is performed in each layer separately. In our approach, we have adopted the same method only to separate layers and to find colour which will be used as a key to search the template which is most similar to the test symbol templates. Here, recognition does not require separate layers to interpret the symbols or toponym. The steps involved in our approach are as given below.

\subsection{Image enhancement}

The training image is enhanced such that the R, G, B values of the pixels are either 0 or 255 . The heuristic or available alternatives are as follows. Pixels with a small difference in their R, G, $\mathrm{B}$ values are set to either fully black $(0,0,0)$ or fully white $(255,255,255)$ depending upon the average intensity value. Otherwise the maximum of the three values is set to 255 and others to 0 . So we have the following classes to represent layers: $\mathrm{C}_{0}=\{0,0,0\}, \mathrm{C}_{1}=\{255,0,0\}, \mathrm{C}_{2}=$ $\{0,255,0\}, C_{3}=\{0,0,255\}$ and $C_{4}=\{255,255,255\}$ for black, red, green, blue and white, respectively. If two values are same and the maximal, both of them are set to 255. So we have three additional classes to represent layers: $C_{5}=\{255,255,0\}, C_{6}=\{0,255,255\}, C_{7}=\{255$, $0,255\}$ for yellow, cyan and magenta, respectively. However, since in our present experiment, selected maps do not have any such colour layers, any pixel satisfying one of these is mapped to one of $C i(i=0,1, \ldots, 4)$ depending on the intensity over its neighbourhood. This allows us to deal with only five classes.

Clusters are group of same colour pixels and so are layers. Here five clusters (black, white, red, and green and blue) result five layers. As white colour cannot be assigned for foreground colour object, four layers are considered for finding symbol. Whatever symbols recognized are the combination of these colours only. (Here, combination does not mean the mixing of RGB; it means single colour, two colour or multi colour objects or symbols). Example, river with rock is two colour symbols. Blue colour is assigned for river or water feature and black colour is for rock or sand in river bed. Our algorithm successfully finds two templates one for water and one for rocks with different colour index i.e., two sub-templates from two layers for same object. Also template generated for river is different from river carrying rocks. Thus, template matching algorithm searches a template with blue colour index in which it finds two templates (we are saying it as two only to illustrate the concept) and then matching is performed with the template found in testing image to determine its type i.e., whether it is river or river with rock.

In this paper, initial image enhancement process is done for determining the initial positions of the centroids of the clusters. K-means algorithm is applied to separate final clusters/layers. Apart from these 5 primary clusters/layers we may also require to separate some secondary layers (yellow, cyan and magenta) and these 8 give us almost complete description of the map. But as said earlier, it is not required in current maps which are under study. 


\subsection{Segmentation}

The K-means algorithm (Chanda \& Dutta Majumdar 2000) is used to generate $K=5$ clusters, where each pixel of the original image is allocated to one of the five clusters such that the intra-class distance is minimum. The feature vector is selected to be the normalized original $\mathrm{R}, \mathrm{G}, \mathrm{B}$ values, along $r_{i}=\frac{R_{i}}{R_{i}+G_{i}+B_{i}} ; g_{i}=\frac{G_{i}}{R_{i}+G_{i}+B_{i}} ; b_{i}=\frac{B_{i}}{R_{i}+G_{i}+B_{i}} ; I_{i}=\frac{R_{i}+G_{i}+B_{i}}{3 \times 255}$ with the normalized intensity $I$ (' $I$ ' was added as a component of the feature vector in order to differentiate between the black and white clusters, as their normalized $\left\{r_{i}, g_{i}, b_{i}\right\}$ values tended to be similar) as follows: The initial cluster centers are supplied as the average of the normalized components $\left(r_{j}, g_{j}, b_{j}, I_{j}\right)$ of the pixels corresponding to each of the $C j$ 's of the enhanced image, where $r_{j}=\frac{1}{n_{j}} \sum_{i \in C_{j}} r_{i}, g_{j}=\frac{1}{n_{j}} \sum_{i \in C_{j}} g_{i}, b_{j}=\frac{1}{n_{j}} \sum_{i \in C_{j}} b_{i}$ and $I_{j},=\frac{1}{n_{j}} \sum_{i \in C_{j}} I_{i}$, where $n_{j}$ is the total number of points in class or group $C_{j}$ of the enhanced image. K-means segmentation treats each symbol or any foreground object as having a location in space (Dhar \& Chanda 2006). We found partitions such that objects within each cluster are as close to each other as possible, and as far from objects in other clusters as possible. K-means segmentation requires that distance metric to quantify how close two objects are to each other. For every object in topographic map input, $\mathrm{k}$-means returns a value corresponding to a cluster. The output from k-means will be used in the template matching.

Once the final cluster centers are determined, the map image is separated into the desired layers. Cluster index is stored in binary file. Templates generated using shape analysis and their respective cluster indices are stored as colour indices in binary file. Colour index helps to narrow down the search space for template matching. For example, if green object is recognized then its contour is matched against templates which are stored with their colour index corresponding to green layer.

\section{Shape analysis}

Shape analysis methods can be classified according to many different criteria. The first criterion is based on the use of shape boundary points as opposed to the interior of the shape. The two resulting classes of algorithms are known as boundary also called external or internal. Many studies are reported to parse the shape boundary (Nevatia 1982; Goshtasby 1985; Davis 1986; Kartikeyan \& Sarkar 1989; Taza \& Suen 1989) and various Fourier transforms of the boundary (Zahn \& Roskies 1972; Granlund 1972; Richards \& Hemami 1974; Persoon \& Fu 1977; Wallace \& Wintz 1980).

Topographic map understanding typically involve two steps: feature extraction in which the patterns are represented by a set of features and classification in which decision rules for separating pattern classes are defined. This section introduces Shape Analysis (SA) which allows finding a symbol on topographic map presented in the form of the exterior outlines. An outline always leads to changes in intensity of an image. A major motivating factor behind the use of shape analysis is a collective understanding about symbol's form, size and orientation can be obtained from an external outline of the shape of symbol.

In the present work, canny edge detection technique is used and resulting edge segments with fixed length and slopes within different ranges are proposed as primitives for shape analysis in describing symbols on a topographic map. This method uses exterior outline to represent symbols. Complex valued chain coding encodes exterior outline and represents exterior spatial information. The illustration of complex numbered chain coding representation of outline is given in figure 1. Each vector of an outline is named as primitive vector (PV) and sequence of 


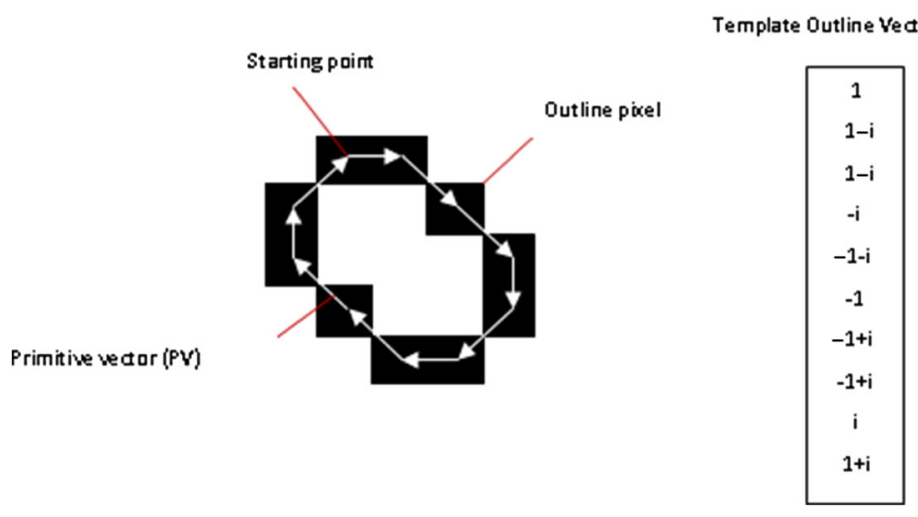

Figure 1. The illustration of complex numbered chain coding representation of outline.

complex-valued numbers are designated as template-outline-vector (TOV). The template outline vector does not depend on the orientation and counter change or interchange of the source image of the symbol. As the outline is encoded relative to starting point, this mode of coding is invariant with respect to the translation of an initial outline. Orientation of symbol on certain angle is equivalent to each PV of an outline on the same angle. The starting point modification conducts to TOV cycle shift. As PVs are encoded concerning the previous point, it is clear that as a modification of starting point, the sequence of a PV will be the same, but the first PV will be what begins at the starting point. The source image of symbol resizing can be considered as multiplication of each PV of an outline to scale factor. As a scalar product,

$$
\eta=(\Gamma, \mathrm{N})=\sum_{n=0}^{k-1}\left(\gamma_{n}, v_{n}\right)
$$

where $\mathrm{k}$ is dimensionality of a TOV, $\gamma_{n}$ is $n^{\text {th }}$ primitive vector of outlines $\Gamma$ of template, $v_{n}$ is $\mathrm{n}^{\text {th }}$ primitive vector of outline $\mathrm{N}$ of test image. $\left(\gamma_{n}, v_{n}\right)$ is the scalar product of complex numbers. In shape analysis, primitive vector of identical dimensionality is considered for the scalar product. That is the number of the primitive vectors in outlines should be same.

\subsection{Normalized scalar product (NSP)}

In a proposed system, a measure of closeness of vectors is provided by $\eta$, normalized scalar product which is defined (Mehtre et al. 1997) as:

$$
\eta=\frac{(\Gamma, \mathrm{N})}{|\Gamma||\mathrm{N}|}
$$

The magnitude of $\Gamma$ and $\mathrm{N}$ i.e., length of outlines calculated as:

$$
|\Gamma|=\left(\sum_{n=0}^{k-1}\left|\gamma_{n}\right|^{2}\right)^{\frac{1}{2}} \quad \text { and } \quad|N|=\left(\sum_{n=0}^{k-1}\left|v_{n}\right|^{2}\right)^{\frac{1}{2}}
$$

Here, elementary vector $\gamma$ and $v$ constitutes primitive vector as $\Gamma=\left(\gamma_{0}, \gamma_{1}, \ldots, \gamma_{k-1}\right)$ and $N=\left(v_{0}, v_{1}, \ldots, v_{k-1}\right)$, respectively. 
The norm of the NSP has upper bound value as 1 if it is equal to product of arbitrary complex number and primitive vector of outline.

$$
\Gamma=\mu \mathrm{N} .
$$

The outline $\mu \mathrm{N}$ means it is the same outline $\mathrm{N}$, but scaled to larger sizes. The size and orientation have been described by the arbitrary complex number $\mu$. The magnitude of the NSP reaches value one, if primitive vectors of two outlines are same, even though oriented at different angles and multiplied by some coefficient. The NSP concept is implemented in automated topographic map understanding system because topographic maps have a number of instances of the same object at various scales and orientation. For example, scalar multiplication of an outline mostly on them, but turned at a certain angle. The normalized scalar product of a vector on itself becomes 1 . If outline turns 90 degrees, then it becomes $0+\mathrm{i}$, and if turn on 180 degrees gives -1 . The properties of the NSP are shown in table 1.

Similarity measure is a function that computes the degree of similarity between two vectors. In the current study, the similarity measure has been implemented as normalized scalar product (Mehtre et al. 1997). The norm of the NSP is calculated using (7) to find maximum similarity between two outline vectors.

$$
\left|N S P_{(\Gamma, \mathrm{N})}\right|=\left(\sum_{n=0}^{k-1}\left|\gamma_{n}\right|^{2}\right)^{\frac{1}{2}}
$$

Here,

$$
\gamma_{n}=\sum_{j=1}^{n}\left(f_{j}^{q}-f_{j}^{i}\right),
$$

where, are discrete Fourier descriptor of query image and original image, respectively.

The NSP is extremely good for search of outlines similar among themselves. But there is one bottleneck problem not allowing it to use directly i.e., starting point selection for chain coding. The normalized value as per (6) is reached only if the starting point of outlines coincides. If outlines are identical but PV reference begins with other starting point then the norm of the NSP is not equal to a unity.

To overcome above limitation, interrelation function (IF) is implemented in this system. Values of this function show outline $\Gamma$ and $\mathrm{N}$ is how much similar if to shift starting point $\mathrm{N}$ on $m$ positions. Interrelation function is defined on all set of PV but as cycle shift on k leads to an initial outline, with phase $\mathrm{k}$. For an example, if $\mathrm{N}=\left(\mathrm{n}_{1}, \mathrm{n}_{2}, \mathrm{n}_{3}, \mathrm{n}_{4}\right), \mathrm{N}^{(1)}=\left(\mathrm{n}_{2}, \mathrm{n}_{3}, \mathrm{n}_{4}, \mathrm{n}_{1}\right)$,

Table 1. Properties of the normalized scalar product of outlines.

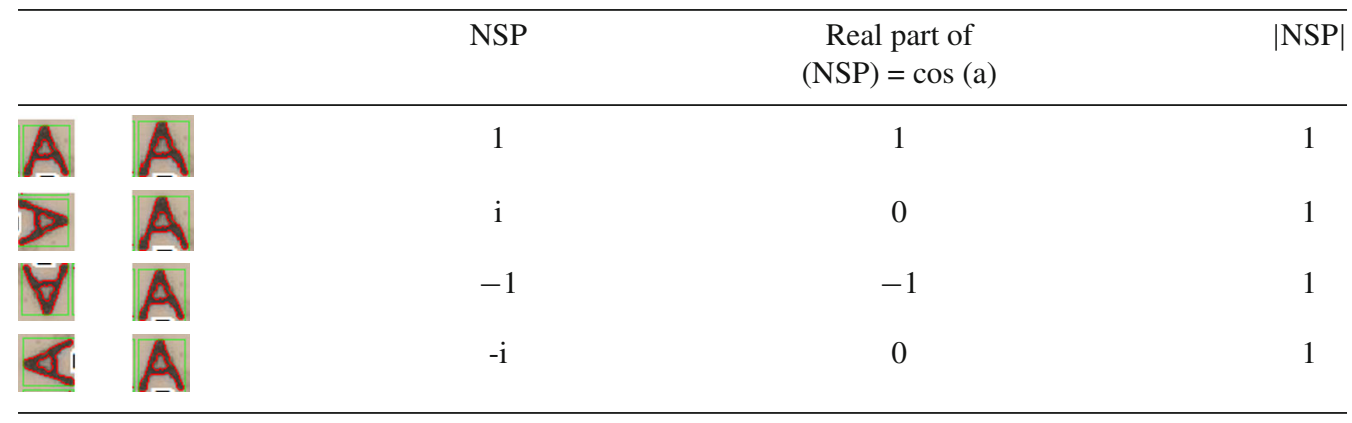


$\mathrm{N}^{(2)}=\left(\mathrm{n}_{3}, \mathrm{n}_{4}, \mathrm{n}_{1}, \mathrm{n}_{2}\right)$ and so on. $\mathrm{N}^{4}$ leads to $\mathrm{N}$ i.e., cycle shift on $\mathrm{k}$ leads to outline itself. Therefore, values of this function are considered only in limits from 0 to $k-1$. The magnitude having the maximum norm among values i.e., interrelations function (IF) value is calculated as:

$$
\tau(m)=\left(\Gamma, N^{(m)}\right), \quad m=0, \ldots, k-1,
$$

where is the outline retrieved from the $\mathrm{N}$ by cycle shift on its PV by $m$ elements.

Then similarity measure,

$$
\tau_{\max }=\max \left(\frac{\tau(\mathrm{m})}{|\Gamma||\mathrm{N}|}\right) .
$$

The $\tau_{\text {max }}$ gives a measure of similarity of two outlines invariant to transposition, scaling, rotation and starting point shift. The magnitude $\tau$ defines a degree of similarity of outlines, and becomes one of similar outlines, and $\operatorname{argument} \arg \left(\tau_{\max }\right)$ shows an angle of orientation of one outline, with reference to another. The list produced is ranked using $\tau_{\max }$ which is used in template matching.

\subsection{Estimation of shape descriptor}

The normalised scalar product (NSP) concept is implemented using shape descriptors and estimated as a Fourier descriptor for discovered outlines which are calculated by (6). The properties of NSP shown in table 1 have been implemented to make system's performance invariant to orientation of symbol or letter or number.

The last vector of an outline always leads to the starting point. Each vector of an outline has been named as a primitive vector (PV) and sequence of complex-valued numbers templateoutline-vector (TOV). Outlines have been described by a vector $\Gamma$, in terms of their primary and basic vectors by $\gamma$ as $\Gamma=\left(\gamma_{0}, \gamma_{1}, \ldots, \gamma_{k-1}\right)$. An operation over an outline of symbol yields a vector of complex valued code which has distinguished mathematical properties. Main motivation behind using a complex valued chain is that it is having resemblance with two-dimensional coding where the outline is specified as a population of the PVs presented in the two-dimensional coordinate reference model. If TOV is increased by some scale then the NSP will become 1 (simply to see from the (6)). The magnitude of NSP remains constant for various instances of symbols subjected to different instances at different transposition, rotation and scaling of outlines. The magnitude of the NSP of outlines becomes one in situation when these two outlines are coinciding. The NSP has been used to search the outlines and extract it from similar patterns. But it is susceptible to a starting point selection for chain coding. The matter is that the specified in (6) is true only if the starting point of outlines coincide. As the chain code has been used for template matching it must be invariant of the selection of starting point. Hence the difference in successive direction, i.e., interrelation has been implemented as an interrelation factor (IF) $\tau_{\max }$. It gives a measure of similarity of outlines after changing the starting point on template from one position to other position. Maximum of IF is invariant to transposition, scaling, rotation of objects and starting point shift on outline while encoding with the complex valued chain coding.

\section{Methodology}

Representation, description, classification and recognition are important tasks in any interpretation system. There are two critical steps in designing the map understanding system. First, 
to represent pattern images in flexible scheme and second is to perform matching on the representational scheme. There are many representation schemes used to describe the pattern image such as list, string, and the graph. In the present study, symbols are represented using a chain code technique which is a more flexible scheme for representation and description of pattern image also. After segmentation of symbols from the background, it is necessary to represent and describe its shape in characteristic features for computer processing during pattern recognition and understanding. Shape descriptor may be either internal or external descriptors depending on whether they encode the boundary of a shape or the part inside the boundary. In the present study, an external representation has been used which focuses on a symbol shape outline feature using the spatial organization of shape region, i.e., external space domain technique. One of the most common external space domain descriptor, boundary chain coding has been used to represent shapes. Most structural matching methods deal with graphical representations and string representations directly as in (Koo \& Yoo 1998) whereas template matching has to be carried out using cross correlation and exhaustive search, i.e., translating the template over every position in the search area (Cox 1995). Template matching provides an effective way to interpret unknown samples by comparing them to a set of known prototype or template with same cluster index. Hence, template matching has been used to identify shapes. In template matching, the test image of the symbol has been matched with the stored prototype or reference pattern. Based on similarity criterion, the test image is interpreted as the prototype or template pattern that is the best match in the input. Top-down strategy has been used for recognition phase. It suggests a strategy in which system starts from the model of symbol and try to fit the model into the template database that is created in training. The flow graph illustrating the main phases in the development of the topographic map letter and number understanding system is shown in figure 2 . The general sequence of an overall operation looks as:

(i) Pre-processing of the image such as smoothing, a noise filtration, contrast raise and enhancement.

(ii) Colour layer separation using k-means algorithm and deriving cluster value of each layer.

(iii) Binarization of the image and selection of outlines of symbols.

(iv) Filtration of the outlines.

(v) Conversion of outlines to uniform length i.e., outlines equalization. (If outlines of the same symbols are not of similar length then equalization is required. Inner product or cross correlation is possible for primitive vectors having uniform dimension.)

(vi) Search for all discovered outlines and searching of the template maximum similar to the given outline templates with same cluster value from binary model.

\subsection{Pre-processing}

The input for topographic map letter/number understanding system is a digital image of the Indian topographic map developed by the Survey of India. To extract data for the shape descriptor formation and matching stages, several steps of pre-processing are required. These include: smoothing, edge detection and generation of boundary chain. In our template matching algorithm, which is based on similarity of primitive $\tau$, the shape of the symbol, letter or number boundaries should be preserved during pre-processing. Artifacts due to badly generated templates would produce errors in the estimation of similarity measures and might result into incorrect recognition. To speed up the template matching process and reduce the number of false boundaries, the number of noise outlines generated in edge detection should be minimized. This is done by applying the smoothing operator to an input map. A canny edge detector is used to find edges in smoothing topomap. In most of the image analysis systems, binarization of the 
scanned gray level image is done prior to further processing. In the proposed method, locally adaptive binarization method is adopted for gray scale form of topographic map with low contrast and variable background intensity (Trier \& Taxt 1995). Canny outlines are dilated for filtering. External boundaries are detected using a chain approximation algorithm. For descriptor estimation boundary data is converted into an ordered code using complex valued chain coding technique. Next, list of templates is obtained using shape analysis methods. For every object in topographic map input, colour separation phase returns value corresponding to a cluster. The cluster center obtained from k-means has been used later in the template matching. Also, the value of the cluster containing the objects obtained programmatically and k-means will not return the same cluster value every time. Here we calculated the cluster center value, which contains the mean ' $a *$ ' and ' $b^{*}$ ' value for each cluster.

\subsection{The brief overview of implementation of functions for outline extraction, template generation}

In the present studies, outlines composed of pixel chains are extracted from a digital Indian topographic map via the canny edge detector (Roy et al. 2007) and used as an initial incomplete set of feature constraints. Smoothing a noise reduction technique has been applied for noise suppression in order to preserve the high spatial frequency detail (e.g., sharp edges) in digital topographic map. It has been explicitly implemented to remove noise in the form of isolated pixels of exceptionally low or high pixel intensity. A binarization method using local adaptive thresholding has been applied. The chain approximation method has been used to find outlines. Next, method to find template has been implemented. It also excludes templates inside other templates. The Outlines are created and stored using complex valued chain coding. A template name has been used as the recognized value. Template matching accelerates searching for a template for the given outline in each cluster by its colour/cluster index. Outcome of operation of this contains an initial outline, and the template discovered for the given outline in specified cluster. Besides, template description contains the degree of similarity, angle of orientation, scale of an outline and cluster index, relative to a template. Finally, templates with its description are stored as a templates model in binary file. When system receives the topographic map on an input, it outputs discovered outlines and recognized outlines.

\subsection{Representation and description process}

The outline of the symbol is a chain of points (pixels), separating it from a background. The boundary chain code describes the outline by a unit size segment with orientation. In the proposed system, the outline has been encoded by the sequence consisting of complex numbers. Then, the outline has been scanned in clockwise direction fixing a starting point on it and each vector of offset is noted by a complex number $\mathrm{a}+\mathrm{ib}$, where $\mathrm{a}$ - point offset on $\mathrm{x}$ axis, and $\mathrm{b}-$ point offset on the y axis. An offset has been preserved and noted concerning the previous point.

\section{Algorithm for outline extraction and complex valued chain code description}

Input: Digital Indian topographic map (figures 3a and 4a).

Output: Complex valued chain code of symbols.

(i) Apply k means segmentation method on enhanced topographic map image.

(ii) Derive cluster index and store it as a key for template matching.

(iii) Binarize image using local adaptive thresholding. (figures $3 \mathrm{~b}$ and $4 \mathrm{~b}$ )

(iv) Extract the boundary or outline of the symbol using the canny edge detector. (figures $3 \mathrm{c}$ and $4 \mathrm{c})$.

(v) Remove noise by applying, erode and dilate morphological operations. 


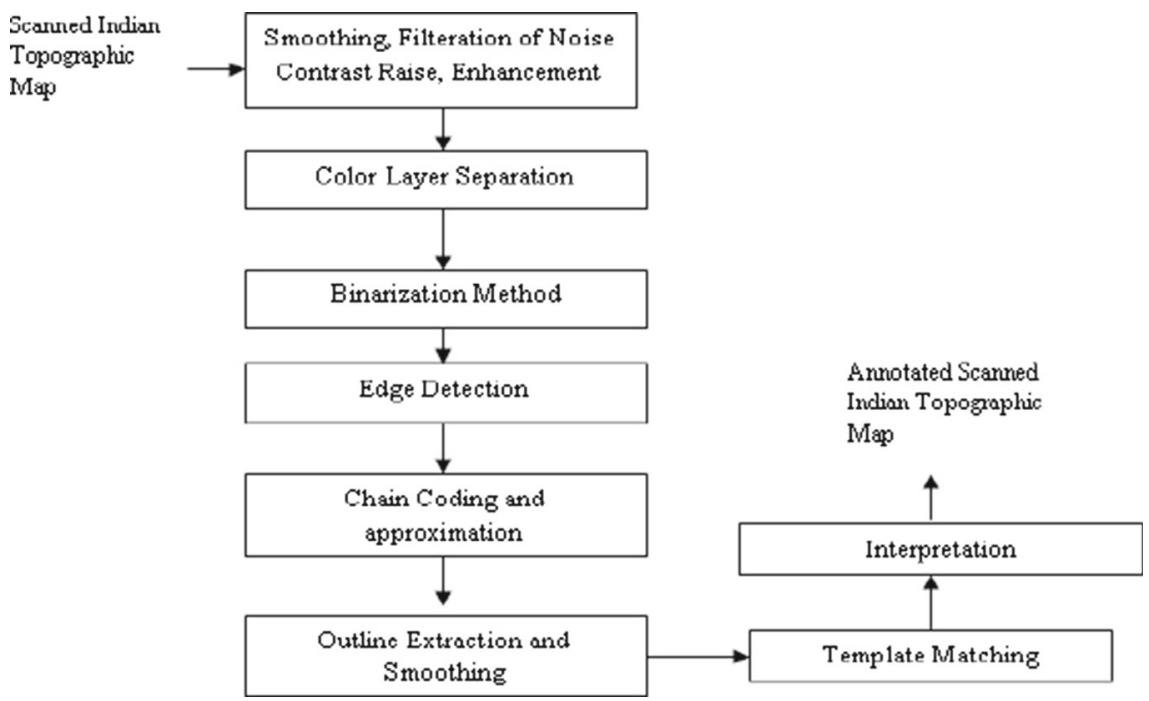

Figure 2. Flow graph illustrating the main phases in the development of the topographic map letter and number understanding system.

(vi) Trace the boundary in clockwise direction and generate 8 directional complex valued chain codes from 1 to $1+i$.

(vii) Compute frequency of the codes.

(viii) Divide frequency of each code by the sum of the frequencies.

(ix) Combine the values in steps (vii) and (viii) to obtain feature vector of the template.

\subsection{Autocorrelation function}

In the present system, an autocorrelation function (AF) has been implemented and is always equal to IF (see (7)) for which $\mathrm{N}=\Gamma$. It is a scalar product of an outline mostly on itself at various shifts of starting point i.e., $m$ defined as below:

$$
v(m)=\left(\Gamma, \Gamma^{(m)}\right), m=0, \ldots, k-1 .
$$

The AF does not depend on a choice of starting point of an outline. If the outline has any symmetry, then its AF has similar symmetry. The norm of an AF is symmetric concerning a central reference $k / 2$ where $k$ is the dimension of TOV. As the AF is the total of pair wise product of a $\mathrm{PV}$ of an outline each pair meets two times at an interval from 0 to $k$. consider the graphics AF for some outlines as in the figures $3 \mathrm{~d}$ and $4 \mathrm{~d}$. The norm the AF is represented by sky blue colour (if $\mathrm{AF}$ is represented only for an interval from 0 to $k / 2$ ). An outline AF is implemented as characteristic descriptions of the shape of an outline. Template and corresponding auto-correlation function for some outlines is shown in figures $3 \mathrm{e}$ and $4 \mathrm{~d}$. From the boundary trace of shape a series of complex numbers are generated. If $\mathrm{N}$ samples of a closed $\Gamma$ are taken, then it is defined by,

$$
u(n) \triangleq x(n)+j y(n), n=0,1, \ldots, N-1
$$




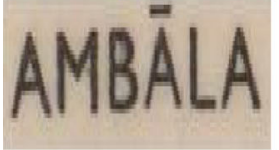

(a)

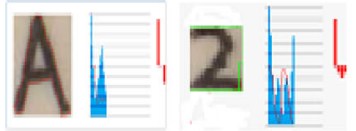

(d)

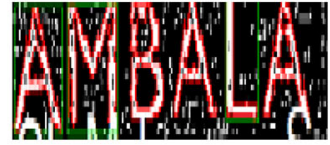

(b)

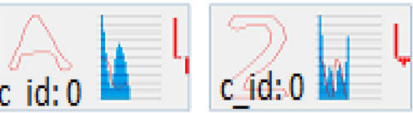

(e)

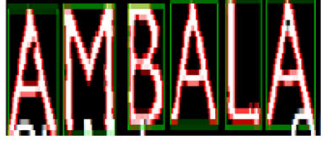

(c)

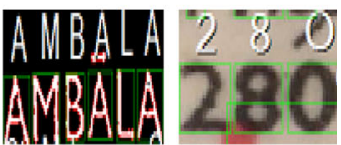

(f)

Figure 3. (a) A part of topographic map. (b-c) Outlines are filtered. (d) Outlines are equalized, in which AF descriptors are calculated. (e) Template matching is performed for the template which is maximum similar to discovered template pattern. (f) Letter and number are interpreted and understood by the system.

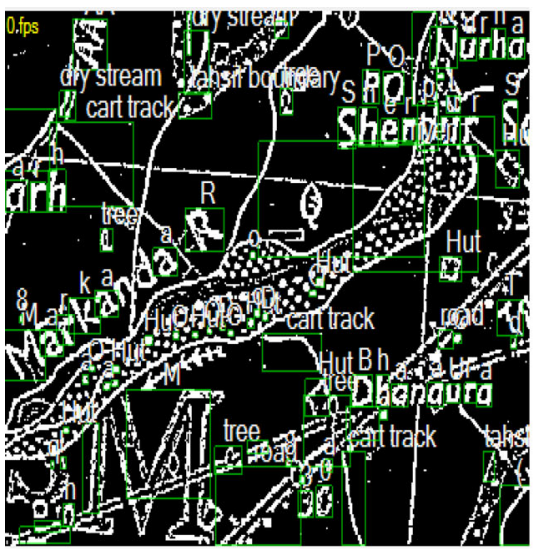

(a)

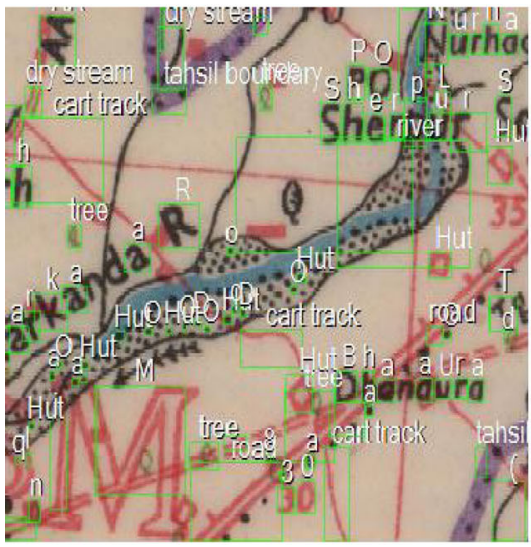

(c)

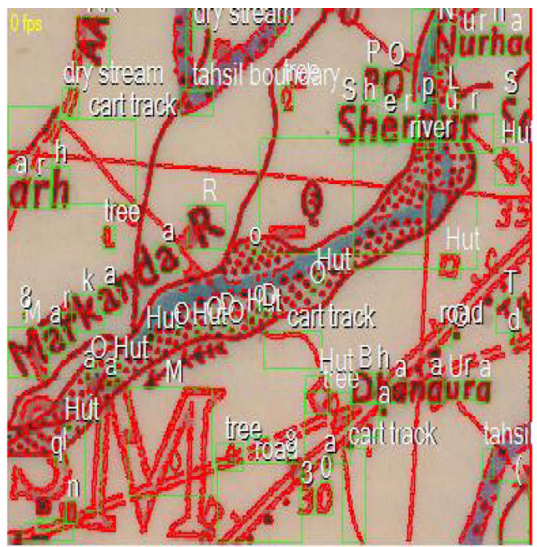

(b)

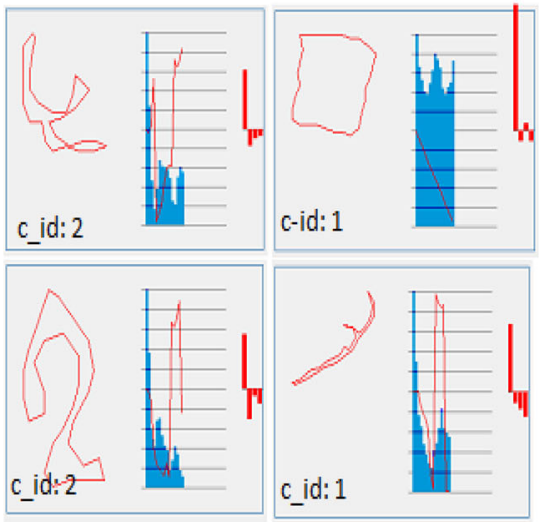

(d)

Figure 4. (a) A gray scale form of a topographic map, (b) Outlines are filtered. (c) Symbol are recognized and understood. (d) Outlines are equalized and AF descriptors are calculated which are used to find a template which matches with the template having same cluster index and most similar to the template pattern discovered. 
Its discrete Fourier transform (DFT) is represented as follows

$$
f(k) \triangleq \sum_{n=0}^{N-1} u(n) \exp \left(\frac{-j 2 \pi k n}{N}\right), 0 \leq k \leq N-1
$$

Discrete Fourier transforms which acts as a descriptor for a series of complex valued chain code has calculated using equation given above.

\subsection{Template matching}

After extracting the shape of symbol or letter or number, next is to match it against a set of template models with same cluster index. However, due to different orientation and interconnectedness within the topographic map, variation within the same type is not uncommon. The proposed study works on template matching, where the templates are formed by chain code. The templates and samples of symbols are digitized images. The degree of similarity between two digital images can be represented using measures of the match. In this approach, correlation measure is used to measure match. For digitized images which are encoded with $\Gamma$ and $\mathrm{N}$, the normalized cross correlation as a measure of a match is defined by the NSP and given by (2) and (3).

Then the Fast Fourier Transform (as given by (11)) has used to calculate the NSP.

The template matching algorithm implemented is as follows:

1. Separate colour layers of test toposheet and derive cluster index.

2. Generate template using algorithm for outline extraction and complex valued chain code description which is described previously.

3. Load the binary model containing all cluster value and set of templates.

4. Extract the shape of test symbol.

5. Derive cluster value.

6. Initialize the level of similarity of primitive to be 1 .

7. Let representative ideal set having cluster value similar to that of test object.

8. Define the measure of match set.

9. If measure of match is not null return match. Otherwise search within representing ideal set having same cluster value.

10. If level of similarity is less than or equal to the maximum primitive level then step 4. In another case exit.

Toponym understanding in the Indian topographic map is illustrated using figure 3 which shows stages in letter and number understanding. Figure 3a shows a part of Indian topographic map. Figure $3 \mathrm{~b}$ shows binarization of crop images by local adaptive threshold, and how the outlines are extracted by Image processing routines. Outlines are filtered that is shown in figure 3c. Figure $3 \mathrm{~d}$ shows how outlines are equalized and AF descriptors are calculated. Figure $3 \mathrm{e}$ shows template, which matches with the template most similar to template pattern discovered. Letters and numbers are recognized and understood by proposed system that is shown in figure $3 \mathrm{f}$. Symbol understanding in the Indian topographic map is illustrated using figure 4 which shows stages in symbol understanding. Figure $4 \mathrm{a}$ shows binarization of crop map image by local adaptive threshold, and how outlines are extracted by image processing routines outlines is filtered that is shown in figure $4 b$. 
Symbols are recognized and understood that is shown in figure 4c. Figure $4 \mathrm{~d}$ shows how outlines are equalized and AF descriptors are calculated and to find a match with the template most similar to the template pattern discovered.

\section{Experimental results}

The system is trained with 150 samples of each of 20 types of symbols and 120 samples of characters and numbers forming toponym from National digital topographic database (NTDB) for OSM (Open Series Map) of Indian topographic maps. The developed system is tested for 200 samples of each type of symbol from NTDB. The small parts of a toposheet image of sizes $350 \times 300,800 \times 600,1500 \times 400$, and 2700X2900 scanned at 300dpi were taken for both training and testing. Let the image already binarized and on it outlines are selected. As further we will work only with points of outlines, we estimate their general amount on the image. For example, we take the maps of size $n^{*} n$ pixels. Then breed its uniform grid with a step $s$. The total length of all grid lines is: $\mathrm{L}=2 \mathrm{n}^{2} / \mathrm{s}$ with this we are able to work with all pixels of images irrespective of, how many objects on it are represented. The complex valued chain code was computed for all the images in the training set by performing chain approximation algorithm described in section 5 . Then, the recognition of the symbols was carried out using a template matching algorithm. In order to analyse how method will perform in practice, five-fold cross validation technique was used. The images were grouped into five-fold cross validation. Each iteration was carried out with one subset as a test set and the rest of the subsets have been combined to form training set. The result is shown in table 2 . An overall recognition rate of $84.68 \%$ is achieved.

It is found that tree, antiquities, broken land and grass is having a higher recognition rate as compared to the rest due to their distinctive shape. The recognition error rate is higher for hut, road, river, river with rock, tank as we generated template for them are not always consistent and often intruded by other features resulting in variant templates at each instance. The chain approximation algorithm results in external spatial representation of symbols. The template generator creates template for each symbol and computes AF descriptor for the matching process. As an AF descriptor (represented by sky blue colour) values (see figure 4d) distributed and represented only for an interval from 0 to $\mathrm{k} / 2$, it is clear that if the exterior boundary has symmetry then its AF leads to symmetry.

Here, $\mathrm{k}$ is the dimension of template-outline-vector. The AF value of hut symmetrical and matches with many anti-pattern as well as template generated from small dot. So, the main power, tele-lines, dry wells on topographic map recognized incorrectly as hut or vice versa. Also, road, river, cart-track and many such objects whose shape cannot be defined by distinctive boundary always lead in incorrect recognizing. All these are the line features represented by irregular shape and size but distinct colour. Shape analysis is insufficient to define its peculiarity in shape. Also, template matching method gives results with maximum matches of AF. Thus, inductively trained system may misinterpret symbol 'river with rock' as a 'river'. For such objects recognition rate is in between $80-90 \%$. As training and testing images are taken from 300 dpi scanned topographic map, all symbols are not isolated, but suffered from overlapping with other symbols hence incorrect interpretation occurred. To evaluate the implementation, the symbol image dataset with two categories is segmented i.e., in the training set and a test set of images. The system is trained with the image from the training set. The learnt template patterns are stored in the binary model. The trained models are used to recognize symbols appeared in training as well as testing image with an overall success rate of $84.68 \%$. The system described in this paper was trained and tested for two independent sets of 8 samples of each of 0 to 9 
Table 2. Recognition rate for symbols in Indian topographic map.

\begin{tabular}{|c|c|c|c|c|c|c|c|}
\hline \multirow[b]{2}{*}{ Symbols } & \multirow{2}{*}{$\begin{array}{c}\text { Conventional } \\
\text { symbols/legends }\end{array}$} & \multicolumn{5}{|c|}{ Five-fold cross validation } & \multirow{2}{*}{$\begin{array}{l}\text { Overall } \\
\text { recognition }\end{array}$} \\
\hline & & Fold-1 & Fold-2 & Fold-3 & Fold-4 & Fold-5 & \\
\hline Hut:Temporary;Permanent & 모 & 87.90 & 93.04 & 82.30 & 82.67 & 86.00 & 86.38 \\
\hline Tree & & 100.00 & 92.89 & 96.03 & 100.00 & 100.00 & 97.78 \\
\hline Grass & & 90.83 & 92.90 & 89.00 & 91.45 & 91.58 & 91.15 \\
\hline Antiquities & & 92.56 & 100.00 & 100.00 & 93.01 & 92.85 & 95.68 \\
\hline Road & $=$ & 86.90 & 92.90 & 90.54 & 82.00 & 91.78 & 88.82 \\
\hline Dry stream & & 90.76 & 90.70 & 89.87 & 91.98 & 87.99 & 90.26 \\
\hline Cart-track & & 89.87 & 90.77 & 91.45 & 92.78 & 89.02 & 90.77 \\
\hline River & & 90.67 & 88.43 & 91.32 & 90.70 & 87.90 & 89.80 \\
\hline Broken-land & $\Rightarrow 2$ & 92.30 & 90.54 & 92.89 & 96.03 & 100.00 & 94.35 \\
\hline Well & & 89.02 & 82.67 & 89.00 & 86.90 & 89.87 & 87.49 \\
\hline Tahsil-boundary & & 82.66 & 78.90 & 81.78 & 91.78 & 89.00 & 84.82 \\
\hline Canal & $\bar{E}$ & 91.45 & 89.00 & 89.17 & 85.90 & 87.99 & 84.82 \\
\hline Dams:Mesonary;earthwork & 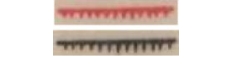 & 87.90 & 86.34 & 92.90 & 90.54 & 88.00 & 89.13 \\
\hline Tube well & & 86.90 & 89.97 & 89.00 & 82.30 & 86.15 & 86.86 \\
\hline Temple & & 91.90 & 92.89 & 92.89 & 90.67 & 91.00 & 91.86 \\
\hline Fort & & 92.60 & 88.43 & 89.87 & 93.02 & 91.45 & 91.07 \\
\hline Church & & 88.90 & 87.00 & 92.30 & 91.11 & 88.92 & 89.64 \\
\hline River with- rock & 283 & 72.67 & 80.05 & 78.45 & 75.00 & 76.01 & 76.43 \\
\hline Tanks & & 86.90 & 83.67 & 81.78 & 86.90 & 82.30 & 84.31 \\
\hline Tower & & 88.78 & 87.05 & 86.90 & 85.56 & 86.90 & 87.03 \\
\hline Overall recognition & & 89.07 & 88.90 & 89.37 & 89.02 & 89.23 & 84.68 \\
\hline
\end{tabular}

numbers with different orientation. Also, the system is trained and tested for 10 samples of each of capital and small A to Z letters in different orientations independently. The result of number recognition is shown in table 3. An overall recognition rate of $92.19 \%$ is achieved for numbers. The result of letters recognition is shown in table 4 . The recognition rate of $91.73 \%$ is achieved for letters. It is found that 2, 3, 4, 5, 6 and 7 is having a higher recognition rate as compared to the rest due to their distinctive shape. The recognition error rate is higher for $0,1,8$, and 9 . The chain approximation algorithm results in external spatial representation of numbers. The template generator creates template for each number and computes AF descriptor for the matching process. The AF value of zero i.e., 0, 1, 8 is symmetrical and matches with other anti-pattern as well as template generated from letters also. So, the letter 'o', 'O', 'i', 'I' on topographic map recognized incorrectly as 0 and 1, respectively. Also, template of 8 and B matches with each other and results misinterpretation. The result of letter recognition is shown in table 4 . Here, 10 samples are taken for each capital and small case but recognition result is not shown separately 
Table 3. Recognition rates of numbers in Indian topographic map.

\begin{tabular}{lcccccc}
\hline \multirow{2}{*}{ Numbers } & \multicolumn{5}{c}{ 5-fold cross validation } & Overall \\
\cline { 2 - 7 } & Fold-1 & Fold-2 & Fold-3 & Fold-4 & Fold-5 & recognition \\
\hline 0 & 90.00 & 88.00 & 87.19 & 82.77 & 82.10 & 86.01 \\
1 & 80.00 & 82.25 & 82.10 & 90.00 & 88.60 & 84.59 \\
2 & 92.92 & 98.03 & 97.64 & 98.03 & 98.41 & 97.00 \\
3 & 98.82 & 98.03 & 95.28 & 99.61 & 98.41 & 98.03 \\
4 & 98.03 & 97.64 & 97.64 & 98.82 & 96.83 & 97.79 \\
5 & 87.19 & 82.10 & 90.00 & 82.25 & 88.00 & 98.03 \\
6 & 96.07 & 97.64 & 95.28 & 98.82 & 95.63 & 96.69 \\
7 & 99.61 & 97.64 & 98.43 & 98.43 & 94.44 & 97.71 \\
8 & 82.25 & 82.10 & 82.77 & 88.19 & 79.21 & 82.90 \\
9 & 82.19 & 85.00 & 79.61 & 82.43 & 86.43 & 83.12 \\
Overall recognition & 90.71 & 90.84 & 90.59 & 91.94 & 90.81 & 92.19 \\
\hline
\end{tabular}

Table 4. Recognition rates of letters in Indian topographic map.

\begin{tabular}{|c|c|c|c|c|}
\hline & \multirow{2}{*}{$\begin{array}{l}\text { Correctly recognized } \\
\text { (20 samples) }\end{array}$} & \multicolumn{2}{|c|}{ Incorrect recognition } & \multirow{2}{*}{$\begin{array}{c}\text { Overall } \\
\text { recognition }(\%)\end{array}$} \\
\hline & & $\overline{\text { Not recognized }}$ & Wrongly recognized & \\
\hline $\mathrm{A} / \mathrm{a}$ & 20 & 0 & 0 & 100 \\
\hline $\mathrm{B} / \mathrm{b}$ & 17 & 1 & 2 & 85 \\
\hline $\mathrm{C} / \mathrm{c}$ & 19 & 0 & 1 & 95 \\
\hline $\mathrm{D} / \mathrm{d}$ & 17 & 0 & 3 & 85 \\
\hline $\mathrm{E} / \mathrm{e}$ & 19 & 0 & 1 & 95 \\
\hline $\mathrm{F} / \mathrm{f}$ & 20 & 0 & 0 & 100 \\
\hline $\mathrm{G} / \mathrm{g}$ & 18 & 0 & 2 & 90 \\
\hline $\mathrm{H} / \mathrm{h}$ & 20 & 0 & 0 & 100 \\
\hline $\mathrm{I} / \mathrm{i}$ & 17 & 1 & 2 & 85 \\
\hline $\mathrm{J} / \mathrm{j}$ & 18 & 0 & 1 & 90 \\
\hline $\mathrm{K} / \mathrm{k}$ & 17 & 1 & 2 & 85 \\
\hline $\mathrm{L} / \mathrm{l}$ & 19 & 0 & 1 & 95 \\
\hline $\mathrm{M} / \mathrm{m}$ & 19 & 0 & 1 & 95 \\
\hline $\mathrm{N} / \mathrm{n}$ & 19 & 0 & 1 & 95 \\
\hline $\mathrm{O} / \mathrm{o}$ & 17 & 0 & 3 & 85 \\
\hline $\mathrm{P} / \mathrm{p}$ & 18 & 0 & 2 & 90 \\
\hline $\mathrm{Q} / \mathrm{q}$ & 17 & 1 & 2 & 90 \\
\hline $\mathrm{R} / \mathrm{r}$ & 17 & 1 & 2 & 90 \\
\hline $\mathrm{S} / \mathrm{s}$ & 19 & 0 & 1 & 95 \\
\hline $\mathrm{T} / \mathrm{t}$ & 18 & 1 & 1 & 90 \\
\hline $\mathrm{U} / \mathrm{u}$ & 20 & 0 & 0 & 100 \\
\hline $\mathrm{V} / \mathrm{v}$ & 18 & 1 & 1 & 90 \\
\hline $\mathrm{W} / \mathrm{w}$ & 20 & 0 & 0 & 100 \\
\hline $\mathrm{X} / \mathrm{x}$ & 19 & 0 & 1 & 95 \\
\hline $\mathrm{Y} / \mathrm{y}$ & 17 & 1 & 2 & 85 \\
\hline $\mathrm{Z} / \mathrm{z}$ & 18 & 1 & 1 & 90 \\
\hline Overall recognition & 477 & 9 & 33 & 91.73 \\
\hline
\end{tabular}


for capital and small case. Letters A, F, H, U, and W show highest recognition rate whereas letters $\mathrm{B}, \mathrm{D}, \mathrm{I}, \mathrm{K}, \mathrm{O}$, and $\mathrm{Y}$ are recognized wrongly with small recognition rate. Many such letters or numbers, whose shape cannot be defined by mere exterior boundary, always lead to incorrect recognizing. Shape analysis using the exterior spatial descriptor is insufficient to define its peculiarity in shape. Also, template matching method gives results with maximum matches of AF. Hence, even though the system is inductively trained for zero gives it as letter 'O'. The letters and numbers touching with other symbols or letters and numbers always yield misinterpretation.

Hence to improve recognition rate, optimal segmentation method need to be researched further. Important achievement is that computational complexity is low. Comparing an AF, generally, does not save us of the necessity of an evaluation an IF. Only the IF states an exact estimation of closeness (i.e., similarity) of outlines. The AF, generally, can coincide for various outlines. But, thus, preliminary selection of templates on an AF essentially narrows down count of candidates on comparing on an IF.

\subsection{Evaluation metrics}

An important criterion for testing the efficiency of the search and retrieval is that the output must include all the similar images (Alvarez 2002).

The list produced is ranked using $\tau_{\max }$. Hence, to evaluate the performance of retrieval, the merit the efficiency of retrieval, $\eta_{T}$ is used. $\eta_{T}=\frac{w}{n_{1}}$, if $n_{1} \leq n 2$; and $\eta_{T}=\frac{w}{n_{2}}$, if $n_{1}>n 2$; where, $w$ is relevant symbol/letter/number which is recognized. Here, $n_{1}$ is $w+x$ and $n_{2}$ is $w+y$.

Variable $x$ is defined as relevant symbol/letter/number but not recognized and $y$ is defined as non-relevant symbol/letter/number but still recognized.

Recall- All the symbol/letter/number recognized by the system, out of the topographic map symbol that is relevant, is called as Recall. Recall is calculated for training (TR) and testing (TS) samples separately as:

Symbol: Recall $(\mathrm{TR})=\frac{w}{n_{1}}=0.9724$ and Recall $(\mathrm{TS})=\frac{w}{n_{1}}=0.944$

Toponym: Recall $(\mathrm{TR})=\frac{w}{n_{1}}=0.8407$ and Recall $(\mathrm{TS})=\frac{w}{n_{1}}=0.80$

Precision- All the symbol/letter/number from topographic map that are recognized by the system, but how many are relevant gives a precision value. Precision is calculated for training (TR) and testing (TS) samples as:

Symbols: Precision $(\mathrm{TR})=\frac{w}{n 2}=0.9745$ and Precision $(\mathrm{TS})=\frac{w}{n 2}=0.8832$

Toponym: Precision $(\mathrm{TR})=\frac{w}{n 2}=0.7971$ and Precision $(\mathrm{TS})=\frac{w}{n 2}=0.7255$

Standard evaluation metrics start with a contingency table (Alvarez 2002). Tables 5 and 6 describe evaluation metrics and the accuracy of the proposed system for symbols and toponym, respectively. The feasibility of the topographic map symbol understanding system has been evaluated for training and testing sample separately. The trained models are used to recognize toponym appeared in training as well as testing image. Outcome of tests is compared, resulting in precision $=0.7971$, recall $=0.8407$ for training samples $(\mathrm{TR})$ and precision $=0.7255$, recall $=$ 0.80 for testing samples (TS).

Symbols: Accuracy $(\mathrm{TR})=\frac{w}{N}=0.9125$, and Accuracy $(\mathrm{TS})=\frac{w}{N}=0.9262$.

Toponym: Accuracy $(\mathrm{TR})=\frac{w}{N}=0.9135$, and Accuracy $(\mathrm{TS})=\frac{w}{N}=0.9629$. 
Table 5. Contingency table for accuracy assessment of symbol understanding.

\begin{tabular}{lcccc}
\hline Symbols & & Training (TR) & Testing (TS) & $\begin{array}{c}\text { Overall accuracy } \\
w /(w+\mathrm{z})\end{array}$ \\
\hline Relevant/correct & Recognized $(w)$ & 2608 & 2360 & $($ Overall $w: 4968$ \\
& Not recognized $(\mathrm{x})$ & 74 & 140 & Overall z: 438$)$ \\
Not relevant/incorrect & Recognized $(\mathrm{y})$ & 68 & 312 & $91.89 \%$ \\
& Not recognized $(\mathrm{z})$ & 250 & 188 & \\
Recall & $w /(w+\mathrm{x})$ & 0.9724 & 0.944 & \\
Precision & $w /(w+\mathrm{y})$ & 0.9745 & 0.8832 & \\
Accuracy & $w /(w+\mathrm{z})$ & 0.9125 & 0.9262 & \\
\hline
\end{tabular}

Table 6. Contingency table for accuracy assessment for toponym understanding.

\begin{tabular}{lcccc}
\hline Toponyms & & $\begin{array}{c}\text { Training (TR) } \\
\text { 260 samples }\end{array}$ & $\begin{array}{c}\text { Testing (TS) } \\
\text { 260 samples }\end{array}$ & $\begin{array}{c}\text { Overall accuracy } \\
\text { w/(w+z) }\end{array}$ \\
\hline Relevant/correct & Recognized $(w)$ & 169 & 156 & (Overall $w: 325$ Overall z: 22) \\
& Not Recognized (x) & 32 & 39 & \\
Not relevant/ & Recognized (y) & 43 & 59 & $93.65 \%$ \\
incorrect & & & & \\
& Not Recognized (z) & 16 & 6 & \\
Recall & $w /(w+x)$ & 0.8407 & 0.80 & \\
Precision & $w /(w+y)$ & 0.7971 & 0.7255 & \\
Accuracy & $w /(w+z)$ & 0.9135 & 0.9629 & \\
\hline
\end{tabular}

Here, $N$ is equal to $w+z$. Variable $z$ is defined as non-relevant symbol/letter/number which is not recognized. Overall percentage of accuracy for symbol is $91.89 \%$. It is calculated as: $(4968 / 5406) * 100=91.89 \%$.

The overall percentage of accuracy for toponym retrieval is $93.65 \%$. It is calculated as: $(325 / 347) * 100=93.65 \%$.

\section{Conclusion}

Topographic maps provide valuable information to a planner or surveyors, but their understanding remains a time-consuming and subjective task. Extraction of information from Topographic map through digitization or vectorization requires human-computer interaction. However, the resulting product is not intelligent enough to handle automated map analysis. The shape analysis method reported in this research work provides a new representation and a description paradigm. Structural approach applied to pre-processed map provides a flexible scheme to represent and describe shapes of boundary outlines using complex valued chain coding. The shape encoding has been constructed to consider orientation and starting point selection criteria. Also, interrelation function and autocorrelation function are implemented for searching outlines similar among themselves and to overcome difficulties with the normalized scalar product. Template matching has been implemented based on similarity measure which is having less computational complexity. If the base of $t$ number of templates store their AF, searching of a template for a outline of length $k$, by comparing the AF, makes $\mathrm{O}\left(k^{2} t\right)$ which is good estimation. Testing method of 
system yields $84.68 \%$ recognition rate of the symbols of an Indian topographic map. Also, accuracy of retrieval is $91.89 \%$. The recognition rates of letters and numbers are 91.73 and $92.19 \%$, respectively. The overall percentage of accuracy for toponym retrieval is calculated as $93.65 \%$. While the performance of the Indian topographic map understanding system described here is promising, the fully automatic understanding of digital raster maps remains an open problem due to the complexity, wide variability of the characteristics and heavy interconnectedness of different features of topographic maps. The future scope of the study will be to address the specified problems as well as better segmentation and recognition scheme to develop learning and reasoning capacity in the system. It will provide comprehensive solution to understand complete topographic map irrespective of the complexity and wide variation.

\section{References}

Alvarez S A 2002 An exact analytical relation among recall, precision, and classification accuracy in information retrieval. Technical report BCCS-02-01, Computer Science Department, Boston College

Anders K H and Fritsch D 1996 Automatic interpretation of digital maps for data revision. Int. Arch. Photogramm. Remote Sens. 31(Part B4): 90-94

Biederman I and Ju G 1988 Surface vs. edge-based determinants of visual recognition. Cogn. Psychol. 20(1): 38-64

Butenuth M, Gosseln G, Tiedge M, Heipke C, Lipeck U and Sester M 2007 Integration of heterogenous geospatial data in a federated database. ISPRS J. Photogramm. Remote Sens. 62(4): 328346

Chaing Y, Knoblock C and Chen C 2005 Automatic extraction of road intersections from raster maps. GIS'05, In: Proceedings of the 13th annual international workshop on geographic information systems, 267-276

Chan K-F and Yeung D-Y 1999 Recognizing online handwritten alphanumeric characters through flexible structural matching. J. Pattern Recog. Soc. Pattern Recog. 32: 1099-1114

Chanda B and Dutta Majumdar D 2000 Digital image processing and analysis. New Delhi: Prentice Hall

Chen L H and Lieh J R 1990 Handwritten character recognition using a 2-layer random graph model by relaxation matching, 23(9): 1189-1205

Chen H and Walter V 2009 Quality inspection and quality improvement of large spatial datasets, In: GSDI 11 World Conference: Spatial Data Infrastructure Convergence: Building SDI Bridges to Address Global Challenges

Cox G S 1995 A review: template matching and measures of match in image processing

Davis L 1986 Two-dimensional shape representation. In: I Young, K S Fu, L Davis, editors. Academic Press, 233-245

Den Hartog J, ten Kate T and Gebrands J 1996 Knowledge based segmentation for automatic map interpretation. Graphics recognition: methods and applications, Notes in Computer Science 1072, Berlin: Springer, 159-178

Dhar D B and Chanda B 2006 Extraction and recognition of geographical features from paper maps. Int. J. Doc. Anal. Reconit. 8(3): 232-245

Ebi N, Lauterbach B and Anheier W 1994 An image analysis system for automatic acquisition from coloured scanned maps. Mach. Vis. Appl. 7(3): 148-164

Ejiri M, Kakumoto S, Miyatake T, Shimada S and Ichikawa T 1984 Automatic recognition of design drawings and maps, In: Proceedings of the seventh international conference on pattern recognition, 1296-1305

Gader P, Forester B, Ganzberger M, Gillities A, Mitchell B and Yocum T 1991 Recognition of handwritten digits using template and model matching. Pattern Recog. 24(4): 421-431 
Gamba P and Mecocci A 1999 Perceptual grouping for symbol chain tracking in digitized topographic maps. Pattern Recog. Lett. 20(3): 355-365

Goshtasby A 1985 Description and descrimination of planar shapes using shape matrices. IEEE Trans. PAMI 7: 738-743

Graeff B and Carosio A 2002 Automatic interpretation of raster-based topographic maps by means of queries. In: FIG XXII International Congress. Washington, DC (Published on CD-ROM). http://www. fig.net/pub/fig_2002/Ts3-10/TS3_10_graeff_carosio.pdf (accessed 11.01.11)

Granlund G H 1972 Fourier preprocessing for hand print character recognition. IEEE Trans. Comput. 21: 195-201

Heinzle F and Sester M 2004 Derivation of implicit information from spatial data sets with data mining. Int. Arch. Photogramm. Remote Sens. 35(Part B4): 335-340

Heinzle F, Sester M and Anders K H 2005 Graph-based approach for recognition of patterns and implicit information in road networks, In: Proceedings of 22nd International Cartographic Conference, La Coruña, Spain, 9-16

Heinzle F, Anders K H and Sester M 2007 Automatic detection of pattern in road networks-methods and evaluation. International Archives of the Photogrammetry, Remote Sensing and Spatial Information Sciences 36(Part 4/W45). http://tiny.cc/FEyb7

Heutte L, Moreau J V and Lecourtier Y 1993 A new feature extraction strategy for handwritten pseudocharacter recognition based on multiple feature extractors, In: Proc. 6th IGS International Conference on Handwriting and Drawing, Paris, 186-188

Kartikeyan B and Sarkar A 1989 Shape description by time series. IEEE Trans. PAMI 11: 977-984

Kasturi R and Alimony J 1988 Information extraction from images of paper based maps. IEEE Trans. Softw. Eng. 14(50): 671-675

Khotanzad A and Zink E 2003 Outline line and geographic feature extraction from USGS colour topographical paper maps. IEEE Trans. Pattern Anal. Mach. Int. 25(1): 18-31

Kimura F and Shridhar M 1991 Handwritten numerical recognition based on multiple algorithms. Pattern Recog. 24(8): 969-989

Kjersti A, Line E and Tove A 1995 Text Recognition from Gray Level Images Using Hidden Markov Models. Comput. Anal. Images Patterns 970: 503-508

Koo J H and Yoo S I 1998 A structural matching for two-dimensional visual pattern inspection. IEEE Int. Conf. Syst. Man Cybern. 5: 4429-4434

Lam L and Suen C Y 1988 Structural classification and relaxation matching of totally unconstrained handwritten zip code numbers. Pattern Recog. 21(1): 19-31

Luscher P, Weiberl R and Mackaness A 2008 Where is the terraced house? On the use of ontologies for recognition of urban concepts in cartographic databases, In: Ruas, A, Christopher, G (eds.), Headway in spatial data handling. In: Lecture Notes in Geoinformation and Cartography. Springer, Berlin, Heidelberg, 449-466

Mackaness W and Edwards G 2002 The importance of modelling pattern and structure in automated map generalization, In: Proceedings of Joint Workshop on Multi-Scale Representations of Spatial Data, Ottawa, Canada DOI:10.1.1.91.1311\&rep=rep1\&type=pdf

Malerba D, Esposito F, Lanza A, Lisi F and Appice A 2003 Empowering a GIS with inductive learning capabilities: the case of INGENS. Comput. Environ. Urban Syst. 27(3): 265281

Mehtre B M, Kankanhalli M S and Lee W F 1997 Shape measures for content based image retrieval: a comparison. Inform. Process. Manag. 33(3): 319-337

Myers Mulgaonkar G P, Chen C, DeCutins J and Chen E 1996 Verification-based approach for automated text and feature extraction. Graphics recognition: methods and applications, Lecture Notes in Computer Science 1072, Berlin: Springer 1996 190-203

Nevatia R 1982 Shape analysis and recognition. chapter 5, 61-89. Prentice Hall

Nikam G G and Ghosh J K 2012 A map legend understanding system. Proceedings of the International Conf. SocProS 2011, AISC, 131: 41-47 
Palvlidis T 1980 Structural descriptions and graph grammars, in pictorial information system. New York: Springer

Persoon E and Fu K 1977 Shape discrimination using fourier descriptors. IEEE Trans. SMC 7: 170-179

Reiher E, Li Y, Donne V D, Lalonde M, Hayne C and Zhu C 1996 A System for efficient and robust map symbol recognition, In: Proceedings of ICPR'96, IEEE, 783-787

Richards C W and Hemami H 1974 Identification of three-dimensional objects using fourier descriptors of the boundary curve. IEEE Trans. SMC 4: 371-378

Roy P, Liados J and Pal U 2007 Text/graphics separation in colour maps. ICCTA'07, In: Proceedings of the International Conference on Computing: Theory and Applications, 545-551

Samet H and Soffer A 1994 A legend driven geographical symbol recognition system, In: Proceedings of the twelfth International conference on Pattern Recognition, Vol. 2, 350-355

Samet H and Soffer A 1996 MARCO: map retrieval by content. IEEE Trans. Pattern Anal. Mach. Intell. 18(8): 783-798

Samet H and Soffer A 1998 MAGELLAN, Map acquisition of geographical labels by legend analysis. Int. J. Doc. Anal. Recog. 1(2): 89-101

Sezgin T M and Davis R 2005 HMM-based efficient sketch recognition, In: Proceedings of the International Conference on Intelligent User Interfaces. IUI'05. ACM Press, 281-283

Starr L E 1984 Computer assisted cartography research and development report. International cartography association

Steinhauer J H, Wiese T, Freksa C and Barkowsky T 2001 Recognition of abstract regions in cartographic maps, In: Proceedings of the International Conference on Spatial Information Theory: Foundations of Geographic Information Science, 306-321

Taza A and Suen C 1989 Discrimination of planar shapes using shape matrices. IEEE Trans. SMC 19: $1281-1289$

Trier O D and Taxt T 1995 Evaluation of binarization methods for document images. IEEE Trans. Pattern Anal. Mach. Int. 17(3): 312-315

Trier O D, Jain A K and Taxt T 1996 Feature extraction methods for character recognition - a survey. Pattern Recog. 29(3): 641-662

Ventura A D and Schettini R 1994 Graphic symbol recognition using a signature technique, In: Proceedings of the 12th International Conference on Pattern Recognition (ICPR'94), Jerusalem, Israel, 2. IEEE Computer Society, Los Alamitos (CA), 533-535

Viglino J M and Pierrot-Deseilligny M 2003 A vector approach for automatic interpretation of the french cadatral map, In: Proceedings of the Seventh International Conference on Document Analysis and Recognition. ICDAR'03, 304-309

Volz S and Walter V 2006 Linking different geospatial databases by explicit relations. Geoinform. Sci. J. 6(1): 41-49

Wallace T and Wintz P 1980 An efficient three-dimensional aircraft recognition algorithm using normalized fourier descriptors. Comput. Graph. Image Process 13: 99-126

Walter V 2004 Object-wise classification of remote sensing data for change detection, In: Heipke C, Pakzad $\mathrm{K}$, Willrich F (eds.) Integration of Geodata and Imagery for Automated Refinement and Update of Spatial Databases. ISPRS J. Photogramm. Remote Sens. 58(3-4): 225-238

Weindorf M 2002 Structure based interpretation of unstructured vector maps, In: Blostein, D, Kwon, Y (eds.), GREC 2002. In: LNCS, vol. 2390. Springer, Berlin, Heidelberg, 190-199

Wiemann S and Bernard B 2010 Conflation services within spatial data infrastructures, In: 13th AGILE International Conference on Geographic Information Science. Portugal, http://agile2010.dsi.uminho.pt/ pen/ShortPapers_PDF\%5C134_DOC.pdf

Wiskott L and Malsburg C 1993 A neural System for the recognition of partially occluded objects in cluttered scenes- A Pilot Study. Int. J. Pattern Recog. Artif. Int. 7(3): 935-948

Wuersch M and Egenhofer M J 2008 Perceptual sketch interpretation, In: Ruas, A, Gold, C (eds.) The 13th International Symposium on Spatial Data Handling. SDH 2008. Springer, Montpellier, France, 19-38 
Yamada H, Yamamoto K and Hosokawa K 1997 Directional mathematical morphology and reformalized hough transformation for analysis of topographic maps. IEEE Trans. Pattern Anal. Mach. Intell. 15(3): 380-387

Zahn C and Roskies R 1972 Fourier descriptors for plane closed curves. Comput. Graph. Image Process 21: 269-281

Zhang Q 2009 Modeling structure and patterns in road network generalization, In: Workshop on generalisation and multiple representation. Leicester, UK. http://ica.ign.fr/Leicester/paper/ Zhang-v1-ICAWorkshop.pdf 